\title{
Search and investigation of extra-solar planets with polarimetry
}

\author{
H. M. Schmid ${ }^{1}$, J.-L. Beuzit ${ }^{2}$, M. Feldt ${ }^{3}$, D. Gisler ${ }^{1}$, R. Gratton ${ }^{4}$, \\ Th. Henning ${ }^{3}$, F. Joos ${ }^{1}$, M. Kasper ${ }^{5}$, R. Lenzen ${ }^{3}$, D. Mouillet ${ }^{6}$, \\ C. Moutou ${ }^{7}$ A. Quirrenbach ${ }^{8}$, D. M. Stam ${ }^{9}$, C. Thalmann ${ }^{1}$,

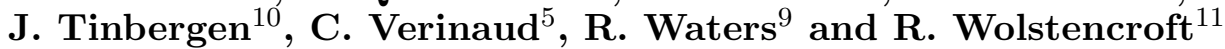 \\ ${ }^{1}$ Institute of Astronomy, ETH Zurich, Switzerland \\ ${ }^{2}$ Laboratoire d'Astrophysique, Observatoire de Grenoble, France \\ ${ }^{3}$ Max-Planck-Institut für Astronomie, Heidelberg, Germany \\ ${ }^{4}$ INAF, Osservatorio Astronomico di Padova, Italy \\ ${ }^{5}$ European Southern Observatory, Garching, Germany \\ ${ }^{6}$ Laboratoire d'Astrophysique, Observatoire Midi-Pyrénées, Tarbes, France, \\ ${ }^{7}$ Laboratoire d'Astrophysique de Marseille, France \\ ${ }^{8}$ Sterrewacht, Leiden University, the Netherlands \\ ${ }^{9}$ Astronomical Institute "Anton Pannekoek", University of Amsterdam, the Netherlands \\ ${ }^{10}$ ASTRON, Dwingeloo, the Netherlands \\ ${ }^{11}$ Astronomy Technology Center, Royal Observatory Edinburgh, UK
}

\begin{abstract}
Light reflected from planets is polarized. This basic property of planets provides the possibility for detecting and characterizing extra-solar planets using polarimetry. The expected polarization properties of extra-solar planets are discussed that can be inferred from polarimetry of "our" solar system planets. They show a large variety of characteristics depending on the atmospheric and/or surface properties. Best candidates for a polarimetric detection are extrasolar planets with an optically thick Rayleigh scattering layer.

Even the detection of highly polarized extra-solar planets requires a very sophisticated instrument. We present the results from a phase A (feasibility) study for a polarimetric arm in the ESO VLT planet finder instrument. It is shown that giant planets around nearby stars can be searched and investigated with an imaging polarimeter, combined with a powerful AO system and a coronagraph at an $8 \mathrm{~m}$ class telescope.

A similar type of polarimeter is also considered for the direct detection of terrestrial planets using an AO system on one of the future Extremely Large Telescopes.
\end{abstract}

Keywords. exoplanets, scattering, polarization, instrumentation.

\section{Introduction}

Direct detection of extra-solar planets from the ground can be achieved with a large telescope, a powerful adaptive optics (AO) system combined with coronagraphy, and a differential detection technique (Angel 2003). A differential detection technique is required because the signal from most planets will be much fainter than the residual AO corrected (and coronagraphic) point spread function (PSF) of the central star. One way to pick out a faint signal of a planet in a strong and variable (speckles!) PSF is to search for spectral features which are present in the planet spectrum but not in the spectrum of the star. Giant planets similar to Jupiter exhibit strong methane bands in the $1.0 \mu \mathrm{m}-1.7 \mu \mathrm{m}$ region which are well suited for differential measurements (see Moutou et al. 2006). An alternative differential method, which is discussed in this paper, is to search for the polarimetric signal from the light reflected by planets. 


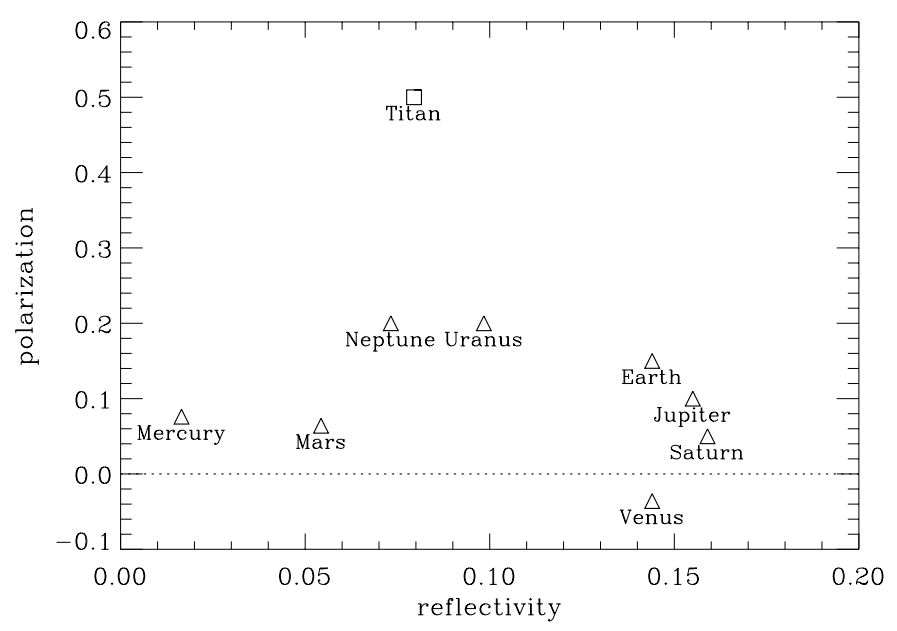

Figure 1. Polarization degree $p\left(90^{\circ}\right)$ versus reflectivity $f\left(90^{\circ}\right)$ for solar system objects at phase angle $\alpha=90^{\circ}$ in the R-band.

Reflected light is the dominant emission component for all cold planets $(<700 \mathrm{~K})$ in the visual to near IR spectral region. This radiation is generally polarized and the degree of polarization is often particularly high at short wavelengths $(<1 \mu \mathrm{m})$ due to Rayleigh scattering by molecules and scattering by haze particles in planetary atmospheres. Thus, the visual-red spectral region is well suited for planet polarimetry.

The search for polarized light from extra-solar planets is very demanding, because the signal decreases rapidly $\propto 1 / d^{2}$ with the orbital separation $d$. Bright, self-luminous, young planets emit predominantly thermal radiation in the near-IR $(>1 \mu \mathrm{m})$ which is not or hardly polarized. Thus, a successful polarimetric detection of extra-solar planets requires strongly polarized $(\gg 1 \%)$ planets and a very sensitive polarimeter in a sophisticated extra-solar planet instrument at a large telescope.

\section{Expected polarization from planets}

The expected polarization of extra-solar planets can be inferred from observations of solar system planets. A crucial parameter for the polarization of planets is the phase angle $\alpha$ (angle: central star - planet - observer). For extra-solar planet observations $\alpha$ will be typically in the range $\alpha \approx 60^{\circ}-120^{\circ}$. Rayleigh scattering, and scattering from rocky/sandy surfaces produce for these phase angles a polarization with an orientation perpendicular to the scattering plane. Other $\alpha$ 's are not suited equally well for extrasolar planet observations because the amount of polarized light from the planet is often strongly reduced and the separation to the central star is small. Fig. 1 gives the degree of polarization $p\left(90^{\circ}\right)$ and reflectivity $f\left(90^{\circ}\right)$ (reflected flux normalized to the irradiated flux) in the R-band $\sim 0.65 \mu \mathrm{m}$ for $\alpha=90^{\circ}$ for solar system objects. The values $p\left(90^{\circ}\right)$ and $f\left(90^{\circ}\right)$ were collected or derived from the literature.

Figure 1 illustrates that there exists a large diversity in polarimetric properties for solar system planets which is discussed here briefly:

Mercury and Mars: The polarization in the R-band is about $p\left(90^{\circ}\right) \approx 5-10 \%$ and the reflectivity is low (Dollfus \& Auriere 1974; Dollfus et al. 1983). This is typical for planets (and smaller bodies) with thin or no atmosphere, like Mercury, Mars, or the Moon, where the light is reflected from the rocky/sandy surface. 
Venus: The reflectivity is high but the polarization $p\left(90^{\circ}\right)$ is low because the light is scattered by high altitude clouds (Coffeen \& Gehrels 1969; Dollfus \& Coffeen 1979). Unlike the other planets $p\left(90^{\circ}\right)$ is negative, or parallel to the scattering plane.

Earth: Compared to the other terrestrial planets in the solar system, Earth has a high polarization $p\left(90^{\circ}\right) \approx 15 \%$ (Dollfus 1957) due to Rayleigh scattering (blue sky). The disk-integrated polarization depends on the cloud coverage together with the exposed areas of oceans, forests, deserts and snow fields.

Jupiter: The polarization is very strong $(\sim 50 \%)$ at the poles where Rayleigh scattering dominates and low $(<10 \%)$ at the equator where the light is mainly reflected from clouds (Smith \& Tomasko 1984). The estimated disk-integrated polarization for Jupiter is about $p\left(90^{\circ}\right)=10 \%$ for equatorial sight lines and somewhat higher for polar sight lines.

Saturn: The polarization pattern is similar to Jupiter with an enhanced polarization at the poles (Tomasko \& Doose 1984). However, the overall polarization level is significantly lower than for Jupiter.

Titan: Saturn's moon Titan has a thick Rayleigh scattering haze layer which produces a very high polarization of $p\left(90^{\circ}\right) \approx 50 \%$ (Tomasko \& Smith 1982).

Uranus and Neptune: A high polarization $p\left(90^{\circ}\right) \approx 20 \%$ is predicted based on groundbased observations of the limb polarization (Schmid et al. 2006; Joos \& Schmid 2006).

The huge variety of polarimetric properties of solar system bodies suggests that polarimetry may play an important role in the future characterization of extra-solar planets.

\section{High precision polarimetric imaging}

Very high precision is required with imaging polarimetry to detect a faint, point-like polarization signal of a planet in the residual seeing halo of a bright star. Aperture polarimetry reached already decades ago a very high precision better than $10^{-5}$ based on fast polarization modulators (e.g. Kemp \& Barbour 1981) and a lock-in or photon counting detector. A similar precision can be achieved with array detectors using the ZIMPOL technique. ZIMPOL (Zurich IMaging POLarimeter) uses also a fast polarization modulator and a special CCD camera performing the on-chip demodulation of the modulated signal (e.g. Povel 1995).

The fast modulator, e.g. a ferroelectric liquid crystal working at a modulation frequency of $1 \mathrm{kHz}$, and a polarizer convert the polarization signal into a fractional modulation of the intensity signal. This intensity modulation is converted back into a polarization signal by a special ZIMPOL CCD camera which measures for each active pixel the intensity difference between the two modulation states. For this every second row of the CCD is masked so that charge packages created in the unmasked row during one half of the modulation cycle are shifted for the second half of the cycle to the next masked row, which is used as temporary buffer storage (the CCD can be equipped with cylindrical micro-lenses which focus the light onto the open CCD rows). After many thousands of modulation periods the CCD is read out within less than one second. The sum of the two images is proportional to the intensity while the normalized difference is the polarization degree of one Stokes component.

Because the measurement is fully differential, systematic error sources are reduced to a very low level. Key advantages of this technique are:

- images for the two opposite polarization modes are recorded practically simultaneously (the modulation is faster than seeing variations),

- both images are recorded with the same pixels,

- there are no differential aberrations between the images for the two opposite polarization due to the atmosphere or the telescope/instrument, 


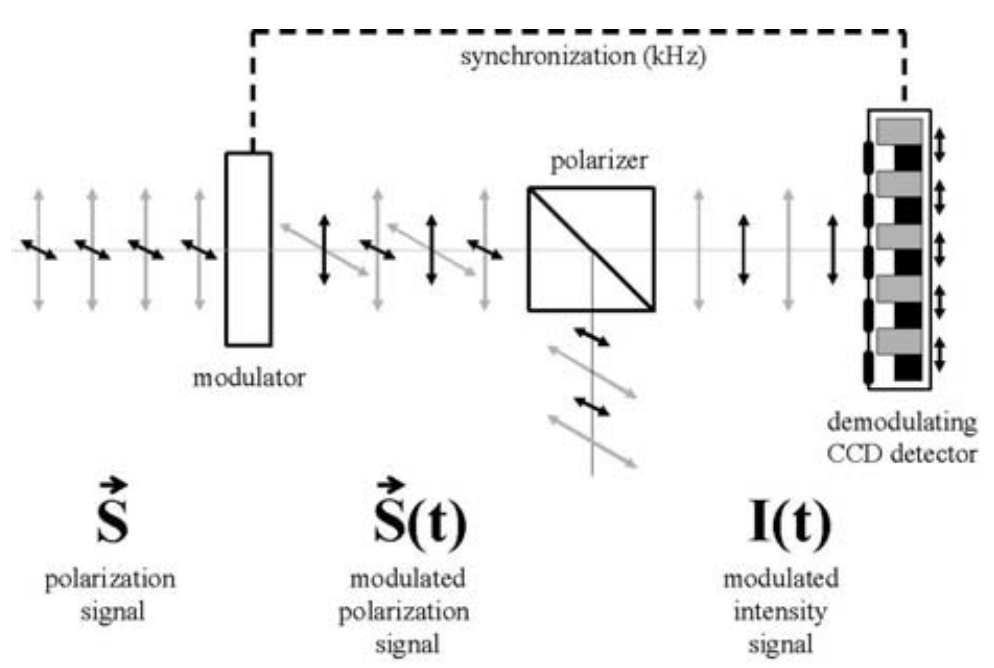

Figure 2. The measuring principle for a fast modulation imaging polarimeter.

- the differential (polarization) signal is not affected by chromatic effects due to telescope diffraction or speckle chromatism.

ZIMPOL has proved to be an extremely precise technique for polarimetric imaging. In solar applications it routinely reaches a precision of better than $10^{-5}$ (e.g. Stenflo \& Keller 1996). For the future one might consider other types of demodulating detectors such as CMOS-detectors (Keller 2004) or avalanche diode arrays.

\section{Implementation into an exo-planet instrument}

ZIMPOL has been considered in combination with an extreme AO system in a feasibility study for the VLT planet finder instrument (Feldt et al. 2003; Gisler et al. 2004). Key requirements are that the entire instrument must be optimized as far as possible for high precision polarimetric imaging. On the other hand the concept for polarimetry must be adapted in order to avoid any disturbing impact on the performance of the AO system, of the coronagraphic system and of the other instruments.

Figure 3 outlines the basic concept for ZIMPOL within the planet finder which will be further investigated in a design study (Beuzit et al. 2006). The imaging polarimeter working in the wavelength range $0.6-0.9 \mu \mathrm{m}$ is located after the AO system, the beam splitters for near-IR instruments and wave front sensor (WFS), and after the coronagraphic system. A polarization switch (e.g. rotatable half-wave plate) is forseen in front of the AO system to reduce and/or calibrate the instrument polarization. The same concept could also be implemented into an extra-solar planet instrument for an ELT (e.g. EPICS concept for OWL - see Verinaud et al. 2006).

\section{Expected polarimetric sensitivity for the VLT and the future ELTs}

An interesting quantity for the polarimetry of extra-solar planets is the polarization contrast, the ratio between polarized flux from the planet to the total flux from the central star:

$$
C_{\mathrm{pol}}=p(\alpha) \cdot C_{\mathrm{flux}}=p(\alpha) \cdot f(\alpha) \frac{R_{p}^{2}}{d_{p}^{2}}
$$




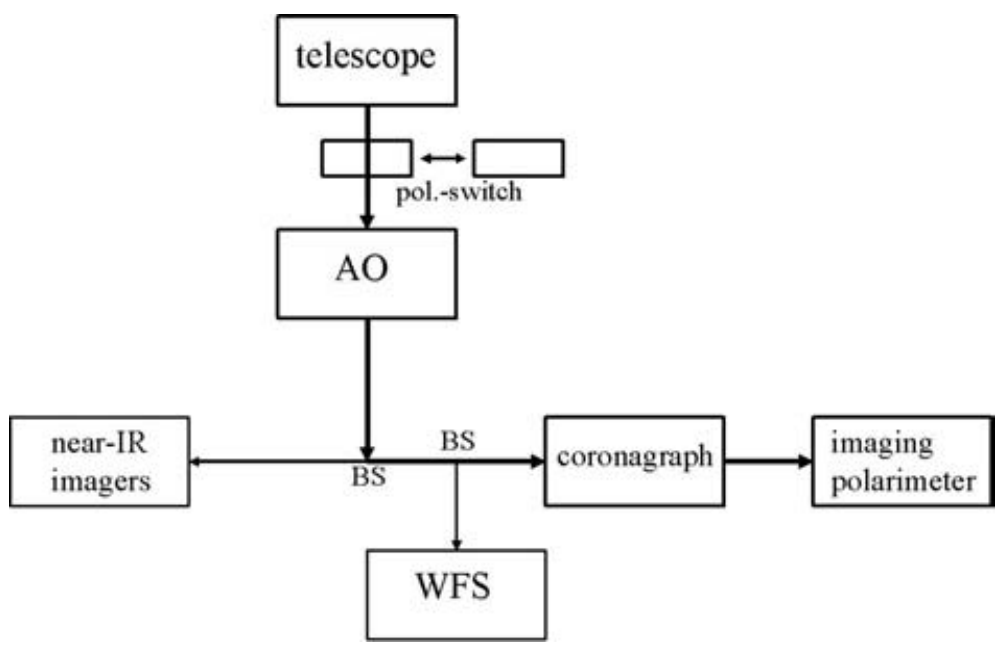

Figure 3. Basic concept of the ZIMPOL polarimeter within the planet finder instrument. The polarization switch is used to reduce and/or calibrate the instrument polarization.

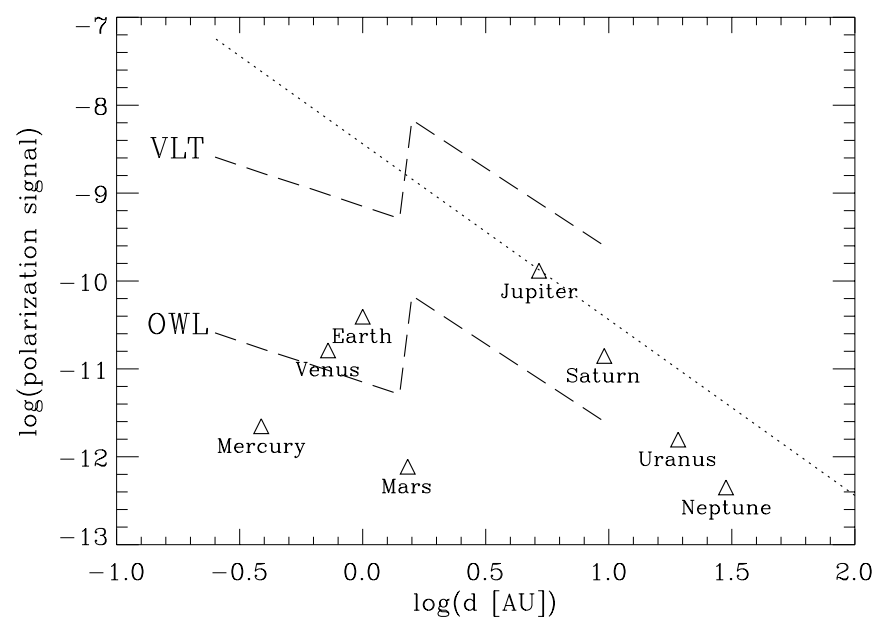

Figure 4. Polarization contrast for solar system planets as function of separation. The dotted line indicates the change in contrast if the separation of Jupiter is changed (but not the other properties). The dashed curves indicate the achievable sensitivity of a planet finder instrument with a band width of $280 \mathrm{~nm}$ at the VLT and an Extremely Large Telescope (OWL) for a solar system analog at 5 pc with an integration time of $4 \mathrm{~h}$.

where $R_{p}$ is the radius of the planet and $d_{p}$ the separation of the planet from the central star. The polarization contrast for the solar system planets (using the reflectivity $f\left(90^{\circ}\right)$ and polarization $p\left(90^{\circ}\right)$ from Fig. 1) is shown in Fig. 4 as function of the separation. Considering the polarization contrast in the R-band, Earth is the second planet in the solar system after Jupiter. The dotted line through Jupiter indicates $C_{\text {pol }}$ if only the separation $d_{p}$ is changed but reflectivity, polarization and radius remain the same.

Also indicated in Fig. 4 are the estimated detection thresholds for planet finder instruments at the VLT or a $100 \mathrm{~m}$ ELT (ESO's overwhelmingly larger telescope OWL) 
for a solar system analog at $5 \mathrm{pc}$ with an integration time of 4 hours. The adopted AO systems have an actuator spacing corresponding to $20 \mathrm{~cm}$, equivalent to about $40 \times 40$ actuators for an $8 \mathrm{~m}$ telescope and $500 \times 500$ actuators for a $100 \mathrm{~m}$ telescope. Achievable Strehl ratios are about $S \approx 0.5-0.7$ depending on the adopted atmospheric conditions, AO control loop frequency and other parameters (see e.g. Chelli 2005). For the imaging polarimeter a precision of $10^{-5}$ is assumed as anticipated for a ZIMPOL system. Due to the ZIMPOL measuring method (see Sect. 3) speckle noise can be neglected.

Figure 4 shows that a Jupiter-like giant planet at 5 pc can be detected with an imaging polarimeter in a planet finder instrument at an $8 \mathrm{~m}$ class telescope if the separation is less than about 2 AU. High polarization $p\left(90^{\circ}\right) \approx 30 \%$ (instead of $10 \%$ as for Jupiter) extra-solar giant planets are also detectable at larger separations with somewhat longer integrations of say 12 hours (instead of 4 hours).

With a planet finder instrument at a $100 \mathrm{~m}$ telescope, Jupiter, Saturn, Earth and Venus could be detectable at 5 pc within 4 hours of integration. Uranus and Neptunetype planets will be very difficult targets even for a $100 \mathrm{~m}$ telescope. Also the polarization signal from atmosphere-less rocky planets (Mercury type) will be very faint and might only be detectable around the very nearest stars $(d<3 \mathrm{pc})$.

These estimates show that polarimetry is a powerful differential technique for the future investigation of scattered light from extra-solar planets from the gound. The polarization signal provides complementary information on the targets when compared to other techniques. Particularly well suited for polarimetric studies are (old) Jupiter-like and Earth-like planets with substantial Rayleigh scattering.

\section{References}

Angel, R. 2003, in: M. Friedlung \& T. Henning, Towards Other Earths: DARWIN/TPF and the Search for Extrasolar Terrestrial Planets, ESA SP-539, 221

Beuzit J.L., et al. 2006, these proceedings

Chelli, A. 2005, A\&A 441, 1205

Coffeen, D.L. \& Gehrels, T. 1969, AJ 74, 433

Dollfus, A. 1957, Suppl. Ann. d'Astrophys. 4, 1

Dollfus, A. \& Auriere, M. 1974, Icarus 23, 465

Dollfus, A. \& Coffeen, D.L. 1970, A\&\&A 8, 251

Dollfus, A., Deschamps, M., \& Ksanfomaliti, L.V. 1983, A\&A 123, 225

Feldt, M., Turatto, M., Schmid, H.M., et al. 2003, in: M. Friedlung \& T. Henning, Towards Other Earths: DARWIN/TPF and the Search for Extrasolar Terrestrial Planets, ESA SP-539, 99

Gisler D., Schmid, H.M., Thalmann, C., et al. 2004, in: A.F.M. Moorwood, \& M. Iye (eds.), Ground-based instrumentation for astronomy, SPIE Conf. Vol. 5492, 1010

Joos, F. \& Schmid, H.M. 2006, these proceedings

Keller, C.U. 2004, in: S. Fineschi, \& M.A. Gummin, Telescopes and Instrumentation for Solar Astrophysics, SPIE Conf. Vol. 5171, 239

Kemp, J.C. \& Barbour, M.S. 1981, PASP 93, 521

Moutou, C., et al. 2006, these proceedings

Povel, H.P. 1995, Optical Eng. 34, 1870

Schmid, H.M., Joos, F., \& Tschan D. 2006, A\&A (submitted)

Smith, P.H. \& Tomasko, M.G. 1984, Icarus 58, 35

Stenflo, J.O. \& Keller, C.U. 1996, Nature 382, 588

Tomasko, M.G. \& Doose, L.R. 1984, Icarus 58, 1

Tomasko, M.G. \& Smith, P.H. 1982, Icarus 51, 65

Verinaud, C., et al. 2006, these proceedings 